\title{
Números cromosómicos de Asteraceae de la Reserva Ecológica del Pedregal de San Ángel (REPSA), México, Distrito Federal
}

\author{
Chromosome numbers in Asteraceae of the Ecological Reserve of the Pedregal de San Ángel \\ (REPSA), Mexico City, Mexico
}

\author{
Fabiola Soto-Trejo ${ }^{1,2 *}$, Guadalupe Palomino ${ }^{1}$ y José Luis Villaseñor ${ }^{2}$ \\ ${ }^{I}$ Laboratorio de Citogenética, Jardín Botánico, Instituto de Biología, Universidad Nacional Autónoma de México. Apartado postal 70-367, 04510 \\ México, D.F., México. \\ ${ }^{2}$ Departamento de Botánica, Instituto de Biologia, Universidad Nacional Autónoma de México. Apartado postal 70-233 , 04510 México, D.F., México. \\ *Correspondencia:fsoto@ibiologia.unam.mx
}

Resumen. Se registraron los números cromosómicos mitóticos o meióticos de 31 especies de Asteraceae en la Reserva Ecológica del Pedregal de San Ángel (REPSA), y por primera vez, los conteos cromosómicos de 3 especies endémicas de México: Acourtia cordata $(2 \mathrm{n}=54)$, Ageratina cylindrica $(2 \mathrm{n}=34)$ y Viguiera buddleiiformis $(\mathrm{n}=17)$. Se discuten los resultados obtenidos de acuerdo con los datos disponibles para cada especie analizada.

Palabras clave: números cromosómicos, Asteraceae, Reserva Ecológica del Pedregal de San Ángel, México, Distrito Federal.

\begin{abstract}
Mitotic or meiotic chromosome numbers are recorded for 31 species of Asteraceae growing in the Ecological Reserve of the Pedregal de San Angel (REPSA), Mexico City. The chromosome counts of 3 species endemic to Mexico are recorded for the first time: Acourtia cordata $(2 n=54)$, Ageratina cilyndrica $(2 n=34)$, and Viguiera buddleiiformis $(\mathrm{n}=17)$. The results are discussed in relation to the available data for each of the analyzed taxa.
\end{abstract}

Key words: chromosome numbers, Asteraceae, Ecological Reserve of the Pedregal de San Ángel, Mexico City, Distrito Federal.

\section{Introducción}

Entre las angiospermas, la familia Asteraceae (o Compositae) es la que mayor diversidad taxonómica presenta con cerca de 1535 géneros y 23000 especies distribuidas en todo el mundo y prácticamente en todos los tipos de vegetación (Bremer, 1994). En México, uno de sus principales centros de diversificación, hay alrededor de 361 géneros y 3021 especies, de las cuales más del 60\% son endémicas (Turner y Nesom, 1998; Villaseñor, 2003; Villaseñor et al., 2004).

Además de su gran diversidad de especies y amplia distribución geográfica, Asteraceae presenta una considerable variación de números cromosómicos y números cromosómicos básicos (el número cromosómico básico (x) representa el número haploide de cromosomas más pequeño en una serie poliploide). En Asteraceae, el número cromosómico más bajo que se ha registrado es $2 \mathrm{n}=4$ en Brachyscome (= Brachycome) dichromosoma-

Recibido: 21 noviembre 2009; aceptado: 04 noviembre 2010 tica C.R. Carter (Carter, 1978; Watanabe et al., 1999) y en Xanthisma gracile (Nutt.) D.R. Morgan et R.L. Hartm. (=Haplopappus gracilis (Nutt.) A. Gray) (Pinkava y Keil, 1977); y el más alto es $2 \mathrm{n}=$ ca. 432 para Olearia albida Hook. f. (Beuzenberg y Hair, 1984). El número cromosómico básico $\mathrm{x}=9$ se propone como ancestral en Asteraceae, pues los grupos basales en la filogenia de la familia, así como las familias más cercanamente relacionadas (Calyceraceae y Goodeniaceae) registran este número básico con mayor frecuencia (Funk et al. 2005; Semple y Watanabe, 2009). En la filogenia de Asteraceae, Funk et al. (2005) reconocen 36 clados; entre ellos se encuentran aquellos con número cromosómico básico de $\mathrm{x}=9,10$ o $11 \mathrm{y}$ otros clados (incluyendo tribus) con números mayores, como $\mathrm{x}=17,18,19,24,25$ o 27 . Además, existe una marcada correlación geográfica entre los clados, encontrándose un número básico de $\mathrm{x}=9$ en clados de América del Sur, $\mathrm{x}=10$ en el Viejo Mundo y $\mathrm{x}=19$ en México y Norteamérica (Funk et al., 2005; Semple y Watanabe, 2009).

La gran variación de números cromosómicos y de números cromosómicos básicos en Asteraceae se atribuye principalmente a eventos de poliploidía, disploidía y aneu- 
ploidía (Solbrig, 1977; Robinson et al., 1981; Stuessy et al., 2004; Semple y Watanabe, 2009).

Existen diversos estudios sobre números cromosómicos para especies de Asteraceae de México (por ejemplo, Turner et al., 1961; Turner y Johnston, 1961; Turner y King, 1964; Turner et al., 1973; Keil y Pinkava, 1976; Sundberg et al., 1986; Keil et al., 1988; Lane y Li, 1993; Strother y Panero, 2001). Sin embargo, hasta la fecha no hay estudios que muestren la variación en los números cromosómicos para alguna flora o hábitat en particular. La información es relevante como apoyo en investigaciones sobre evolución y diversificación en floras y hábitats, y para desarrollar y establecer estrategias adecuadas para la conservación (Kenton, 1986; Martínez y Parker, 1995; Palomino, 2000).

En México hay regiones que por sus características ecológicas, geográficas o geológicas presentan una gran biodiversidad; tal es el caso del Valle de México (Rzedowski, 1954; Rzedowski y Rzedowski 1989, 1993, 2001; Carri1lo, 1995). Sin embargo, debido al rápido e intenso proceso de destrucción y fragmentación de sus ecosistemas, al ser la región del país con mayor presión y afectación por las actividades humanas, solo quedan remanentes en áreas naturales protegidas. Una de estas áreas naturales protegidas es la Reserva Ecológica del Pedregal de San Ángel (de aquí en adelante referida como REPSA), localizada dentro del campus de la Universidad Nacional Autónoma de México, con una extensión de 237.3 hectáreas (Gaceta UNAM, 2005). El tipo de vegetación principal es un matorral xerófilo, denominado por Rzedowski (1954) Senecionetum praecoxis en referencia a la especie dominante Senecio praecox (Cav.) DC. (=Pittocaulon praecox (Cav.) H. Rob. et Brettel). No obstante su pequeña superficie, la REPSA alberga una gran diversidad de plantas vasculares; el recuento más reciente registró 377 especies, $27.8 \%$ de las cuales pertenecen a la familia Asteraceae (Castillo-Argüero et al., 2009). Las investigaciones citogenéticas en plantas de la REPSA son escasas y sólo se han estudiado algunas especies de las familias Cactaceae (como Mammillaria haageana Pfeiff. subsp. elegans D.R. Hunt, registrada con el nombre de Mammillaria sanangelensis Sánchez-Mej., Palomino et al., 1999), Commelinaceae (como Commelina coelestis Willd., C. communis L., C. diffusa Burm. f., Cymbispatha commelinoides (Schult. et Schult. f.) Pichon, Gibasis schiedeana (Kunth) D.R. Hunt y Tradescantia crassifolia Cav., Palomino et al., 1990; Martínez y Palomino, 1997); Fabaceae (como Crotalaria pumila Ortega, Palomino y Vázquez, 1991) y Solanaceae (como Datura stramonium L., Ramírez, 1999). Hasta la fecha, no se ha realizado ningún estudio citológico de especies de Asteraceae de la REPSA, no obstante que esta familia es la de mayor presencia y varias de sus especies son dominantes en la fisonomía del matorral xerófilo.
La falta de información citogenética básica, importante en el mantenimiento y monitoreo de áreas naturales protegidas, así como la destrucción de las comunidades naturales del Valle de México motivaron el presente estudio con el objetivo de determinar el número cromosómico de especies de la familia Asteraceae registradas en la REPSA y así incrementar el conocimiento citológico de su flora.

\section{Material y métodos}

Las especies de Asteraceae estudiadas fueron recolectadas en la REPSA localizada en el sur de la ciudad

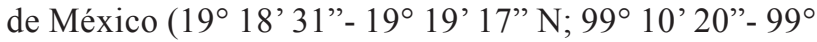
11' 52" O, Fig. 1), en un intervalo altitudinal de 2200 a 2277 m snm (Castillo-Argüero et al., 2004). Durante los años 2005 a 2008 se recolectaron inflorescencias y semillas de diferentes especies; los ejemplares que respaldan las recolecciones fueron determinados y depositados en el Herbario Nacional de México (MEXU) del Instituto de Biología, Universidad Nacional Autónoma de México.

El número cromosómico en mitosis fue determinado a partir de ápices de raíz obtenidos de semillas germinadas en cajas de Petri sobre papel filtro húmedo a temperatura ambiente $\left(18{ }^{\circ} \mathrm{C}\right.$ a $\left.22{ }^{\circ} \mathrm{C}\right)$. Los meristemos radicales fueron pretratados con solución acuosa de 8-hidroxiquinoleína $(0.002 \mathrm{M})$ por 5 horas a 18 ${ }^{\circ} \mathrm{C}$ y fijados en Farmer (3:1 v/v alcohol absoluto: ácido acético) durante 24 horas. Después, el material fue hidrolizado en ácido clorhídrico (1N) durante 15 minutos a $60{ }^{\circ} \mathrm{C}$ y teñido con Feulgen por 15 minutos (García, 1990). Las raíces teñidas fueron procesadas con la técnica de aplastamiento con propiono-orceína al $1.8 \%$.

Para la obtención de cromosomas meióticos, se fijaron in situ inflorescencias inmaduras y se conservaron en Carnoy (6:1:1 v/v/v alcohol absoluto: ácido acético: cloroformo). Las anteras jóvenes fueron analizadas usando la técnica de aplastamiento con propiono-orceína al $1.8 \%$, identificando células en profase y metafase 1 de la meiosis (García, 1990).

Las preparaciones citológicas de cromosomas mitóticos y meióticos se fijaron siguiendo la técnica del hielo seco (Conger y Fairchild, 1953) y se montaron en bálsamo de Canadá. Las mejores metafases fueron fotografiadas con un fotomicroscopio FOMI II Carl Zeiss. La determinación de los números cromosómicos mitóticos y meióticos se basaron en conteos de 5 a 10 células de 3 o más individuos por especie. 


\section{Resultados}

De las 105 especies de Asteraceae registradas en la REPSA por Castillo-Argüero et al. (2009), se estudiaron cerca del 30\%. Los números cromosómicos mitóticos o meióticos se determinaron para 38 muestras que representan 31 especies de diferentes géneros de Asteraceae
(Cuadro 1, Figs. 2-5).

Los números cromosómicos registrados en el presente estudio muestran una amplia variación, desde $2 \mathrm{n}=10$ en Picris echioides L. hasta $2 \mathrm{n}=60$ en Barkleyanthus salicifolius (Kunth) H. Rob. et Brettell y Pittocaulon praecox. De igual manera, los números cromosómicos básicos muestran una gran diversidad $(\mathrm{x}=5,8,9,10,11,12,13,16,17,19,27$

Cuadro 1. Números cromosómicos de especies de Asteraceae del matorral xerófilo de la Reserva Ecológica del Pedregal de San Ángel (REPSA), México, D.F.

\begin{tabular}{|c|c|c|c|c|}
\hline \multirow[t]{2}{*}{ Especie } & \multirow[t]{2}{*}{$\begin{array}{l}\text { Núm. de } \\
\text { colecta }^{a}\end{array}$} & \multicolumn{2}{|c|}{$\begin{array}{l}\text { Número } \\
\text { cromosómico }\end{array}$} & \multirow[t]{2}{*}{ Conteos previos } \\
\hline & & $n$ & $2 n$ & \\
\hline \multicolumn{5}{|l|}{ Tribu Astereae } \\
\hline *Baccharis sordescens DC. & 23,60 & 9 & 18 & n=9 (Powell y Turner, 1963; Keil y Stuessy, 1977) \\
\hline \multicolumn{5}{|l|}{ Tribu Eupatorieae } \\
\hline $\begin{array}{l}* \text { Ageratina cylindrica (McVaugh) R.M. King et } \\
\text { H. Rob. }\end{array}$ & 88 & & 34 & \\
\hline $\begin{array}{l}\text { *Ageratina petiolaris (Moc. et Sessé ex DC.) R.M. } \\
\text { King et H. Rob. }\end{array}$ & 52,82 & 17 & 34 & $\mathrm{n}=17$ (Turner et al., 1962; King et al., 1976) \\
\hline $\begin{array}{l}\text { *Brickellia secundiflora (Lag.) A. Gray var. } \\
\text { secundiflora }\end{array}$ & 46 & & 18 & n= 9 (Turner y King, 1964) \\
\hline Brickellia veronicifolia (Kunth) A. Gray & 55 & & 18 & $\mathrm{n}=9$ (King et al., 1976) \\
\hline $\begin{array}{l}\text { Fleishmannia pycnocephala (Less.) R.M. King et } \\
\text { H. Rob. }\end{array}$ & 173 & 20 & & $\begin{array}{l}n=10(\text { Sundberg et al., 1986) } \\
n=20(\text { Turner et al., 1962; King et al., 1976) } \\
n=30 \text { (King et al., 1976) } \\
n=40(\text { Robinson et al., 1989) }\end{array}$ \\
\hline *Stevia origanoides Kunth & 54 & & 33 & $2 \mathrm{n}=22,2 \mathrm{n}=33,2 \mathrm{n}=44($ Soejima et al. 2001$)$ \\
\hline Stevia salicifolia Cav. var. salicifolia & 33,49 & 12 & 24 & $\begin{array}{l}\mathrm{n}=9(\text { Keil y Stuessy, } 1975) \\
2 \mathrm{n}=24(\text { Zhao y Turner, 1993) }\end{array}$ \\
\hline *Stevia tomentosa Kunth & 149 & & 33 & $2 \mathrm{n}=33$ (Watanabe et al., 1995) \\
\hline \multicolumn{5}{|l|}{ Tribu Heliantheae } \\
\hline Ambrosia psilostachya DC. & 104 & 36 & & $\begin{array}{l}n=18(\text { Keil y Stuessy, 1975) } \\
n=36, n=54 \text { (Payne et al., 1964) }\end{array}$ \\
\hline $\begin{array}{l}\text { Bidens bigelovii A. Gray var. angustiloba (DC.) } \\
\text { Ballard }\end{array}$ & 158 & & 24 & $\mathrm{n}=12($ Olsen, 1980) \\
\hline Bidens odorata Cav. var. odorata & 107 & 12 & & (Keil et al., 1988) \\
\hline Cosmos bipinnatus Cav. & 31,209 & 12 & 24 & $\mathrm{n}=12$ (Melchert, 1968; Carr et al., 1999) \\
\hline
\end{tabular}


Cuadro 1. Continúa

\begin{tabular}{|c|c|c|c|c|}
\hline \multirow[t]{2}{*}{ Especie } & \multirow[t]{2}{*}{$\begin{array}{l}\text { Núm. de } \\
\text { colecta }^{a}\end{array}$} & \multicolumn{2}{|c|}{$\begin{array}{l}\text { Número } \\
\text { cromosómico }\end{array}$} & \multirow[t]{2}{*}{ Conteos previos } \\
\hline & & $n$ & $2 n$ & \\
\hline Cosmos parviflorus (Jacq.) Kunth & 118 & 12 & & n=12 (Melchert, 1968; Carr et al., 1999) \\
\hline Dahlia coccinea Cav. & 05 & & 32 & $2 n=32,2 n=64($ Gatt et al., 1998) \\
\hline Dahlia sorensenii H.V. Hansen et Hjert. & 109 & & 64 & $\mathrm{n}=32$ (Hansen y Hjerting, 1996) \\
\hline Galinsoga parviflora Cav. & 108 & & 16 & $\begin{array}{l}\mathrm{n}=8(\text { Canne }, 1983) \\
\mathrm{n}=16(\text { Lane y Li, } 1993) \\
\mathrm{n}=24(\text { Strother y Panero, } 2001)\end{array}$ \\
\hline *Lagascea rigida (Cav.) Stuessy & 53 & & 34 & $\mathrm{n}=17$ (Stuessy, 1978) \\
\hline *Montanoa grandiflora Alamán ex DC. & 81 & & 38 & $\mathrm{n}=19$ (Solbrig et al., 1972) \\
\hline *Montanoa tomentosa Cerv. subsp. tomentosa & 15 & 19 & & $\mathrm{n}=19$ (Keil et al., 1988) \\
\hline Tithonia tubiformis (Jacq.) Cass. & 18 & & 34 & n= 17 (Keil y Stuessy, 1977) \\
\hline *Verbesina virgata Cav. var. virgata & 47 & & 34 & $\mathrm{n}=17$ (Turner et al., 1961) \\
\hline $\begin{array}{l}* \text { Viguiera buddleiiformis (DC.) Benth. et Hook. } \\
\text { f. ex Hemsl. }\end{array}$ & 143 & 17 & & \\
\hline Zinnia peruviana (L.) L. & 98 & & 24 & $\mathrm{n}=12($ Torres, $1963 ;$ Lane y Li, 1993) \\
\hline \multicolumn{5}{|l|}{ Tribu Lactuceae } \\
\hline Lactuca serriola $\mathrm{L}$. & 91 & & 18 & $\mathrm{n}=9$ (Tomb et al., 1978) \\
\hline Picris echioides L. & 79 & & 10 & (Keil y Pinkava, 1976) \\
\hline Sonchus oleraceus L. & 32 & & 32 & $\mathrm{n}=16($ Turner et al., 1961) \\
\hline \multicolumn{5}{|l|}{ Tribu Mutisieae } \\
\hline$*$ Acourtia cordata (Cerv.) B. L. Turner & 36,67 & 27 & 54 & \\
\hline \multicolumn{5}{|l|}{ Tribu Senecioneae } \\
\hline $\begin{array}{l}\text { Barkleyanthus salicifolius (Kunth) H. Rob. et } \\
\text { Brettell }\end{array}$ & 85 & & 60 & $\begin{array}{l}\mathrm{n}=30 \text { (Turner et al., 1962; Turner y Flyr, } \\
\text { 1966; Powell et al., 1974) }\end{array}$ \\
\hline *Pittocaulon praecox (Cav.) H. Rob. et Brettell & 57 & 30 & 60 & $\mathrm{n}=30($ Strother, 1983$)$ \\
\hline Tribu Tageteae & & & & \\
\hline Dyssodia papposa (Vent.) Hitchc. & 207 & & 26 & $\begin{array}{l}\mathrm{n}=13(\text { Keil et al., } 1988) \\
2 \mathrm{n}=26(\text { Grashoff et al., 1972) }\end{array}$ \\
\hline Tagetes tenuifolia Cav. & 51 & & 24 & $\begin{array}{l}\mathrm{n}=12(\text { Keil et al., } 1988) \\
\mathrm{n}=24(\text { Strother, } 1983)\end{array}$ \\
\hline
\end{tabular}

\footnotetext{
*especie endémica de México; • primer conteo cromosómico para la especie. ${ }^{\text {a }}$ recolectores: F. Soto-Trejo y E. Ortiz-Bermúdez.
} 


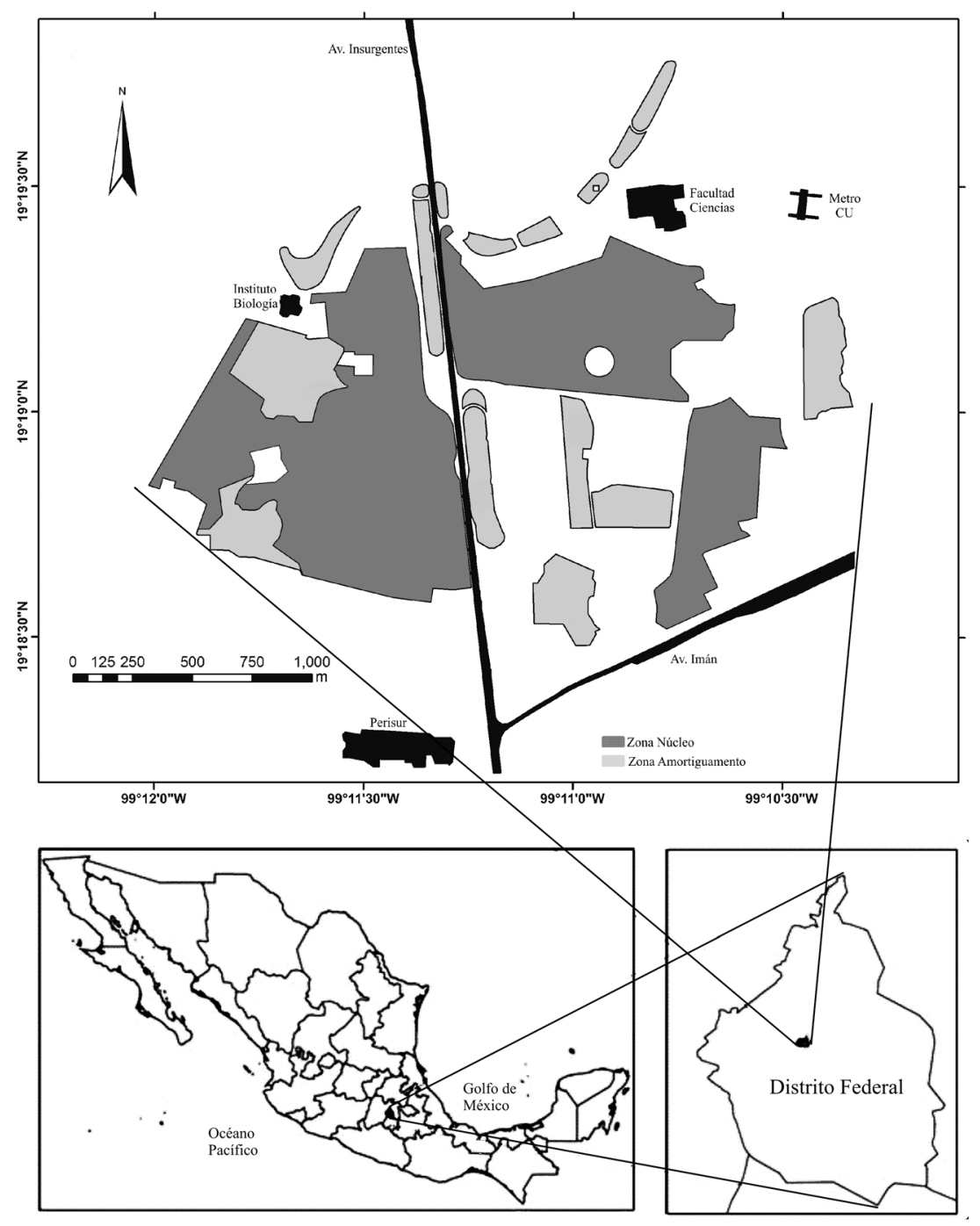

Figura 1. Ubicación geográfica de la Reserva Ecológica del Pedregal de San Ángel (modificado de Gaceta UNAM, 2005).

y 30). Además, el tamaño de los cromosomas varía notablemente a diferentes niveles taxonómicos (entre miembros de distintas tribus, entre miembros de la misma tribu, e inclusive entre miembros del mismo género; véase Figs. 2-5).

Entre estos números cromosómicos destacan los conteos obtenidos por primera vez para 3 especies endémicas de México: Acourtia cordata (Cerv.) B. L. Turner $(2 \mathrm{n}=$ 54), Ageratina cilyndrica (McVaugh) R.M. King et H. Rob. $(2 \mathrm{n}=34)$ y Viguiera buddleiiformis (DC.) Benth. et Hook. f. ex Hemsl. (DC.) $(n=17)$.

\section{Discusión}

Los resultados obtenidos muestran una amplia variedad de números cromosómicos para las especies estudiadas de Asteraceae presentes en la REPSA, reflejando la compleja evolución cromosómica de la familia. El principal mecanismo de evolución cromosómica en Asteraceae es la poliploidía, aunque también son frecuentes la disploidía y la aneuploidía (Solbrig, 1977; Robinson et al., 1981; Stuessy et al., 2004; Semple y Watanabe, 2009). Usualmente, la poliploidía se infiere a partir de los números cromosómicos: una especie poliploide posee 2 o más juegos de cromosomas homólogos y puede ser triploide $(3 \mathrm{x})$, tetraploide $(4 x)$, pentaploide $(5 x)$, hexaploide $(6 x)$, etc. Además, considerando que el número cromosómico básico $\mathrm{x}=9$ es ancestral en Asteraceae, probablemente los números básicos mayores se derivaron de eventos de poliploidía y disploidía (Semple y Watanabe, 2009). 


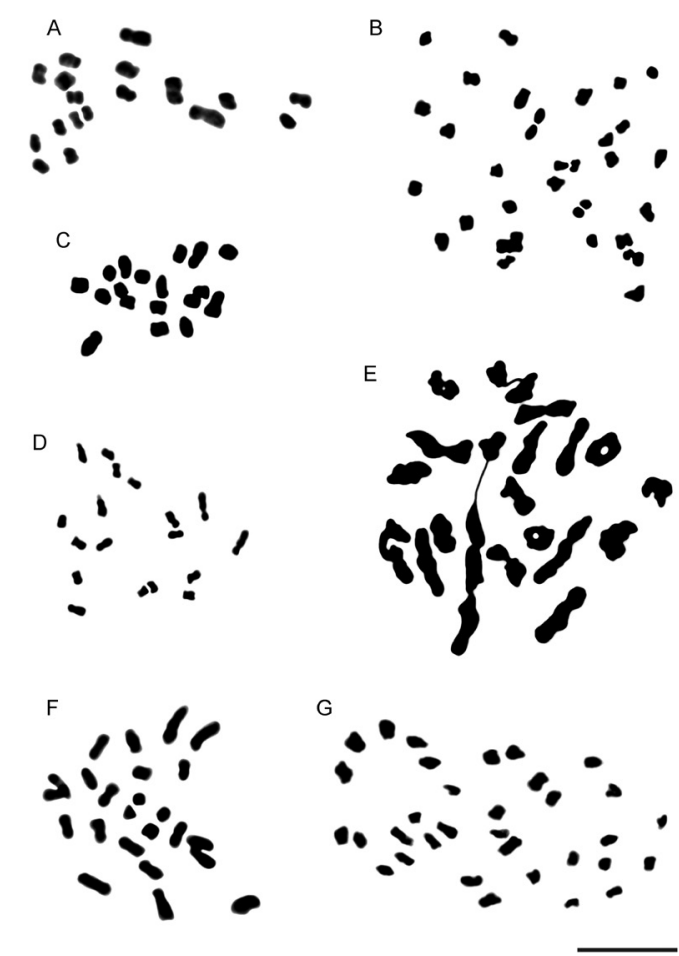

Figura 2. Cromosomas mitóticos (2n) y meióticos (n), de: A) Baccharis sordescens $2 \mathrm{n}=18$; B) Ageratina cylindrica $2 \mathrm{n}=34$; C) Brickellia secundiflora var. secundiflora $2 \mathrm{n}=18$; D) Brickellia veronicifolia $2 \mathrm{n}=18$; E) Fleischmannia pycnocephala $\mathrm{n}=$ 20 II (el símbolo II indica la presencia de bivalentes); F) Stevia salicifolia var. salicifolia $2 \mathrm{n}=24 ; \mathrm{G})$ Stevia origanoides $2 \mathrm{n}=33$. Escala $=10 \mu \mathrm{m}$.

En los siguientes párrafos se discuten por tribu los resultados que se obtuvieron en relación con estudios previos y la información citológica disponible para cada especie.

\section{Tribu Astereae.}

Los conteos cromosómicos $\mathrm{n}=9$ y $2 \mathrm{n}=18$ (Fig. 2A) para Baccharis sordescens DC. coinciden con los conteos previos registrados por Keil y Stuessy (1977).

\section{Tribu Eupatorieae.}

El número cromosómico $2 \mathrm{n}=2 \mathrm{x}=34$ determinado para Ageratina petiolaris (Moc. et Sessé ex DC.) R.M. King et H. Rob. confirma el número que previamente determinaron King et al. (1976); en esta investigación se observó el mismo número para $A$. cylindrica $(2 \mathrm{n}=34$; Fig. $2 \mathrm{~B})$, el cual se registra por primera vez para esta especie. Los números en las 2 especies, ambas endémicas de México, apoyan el

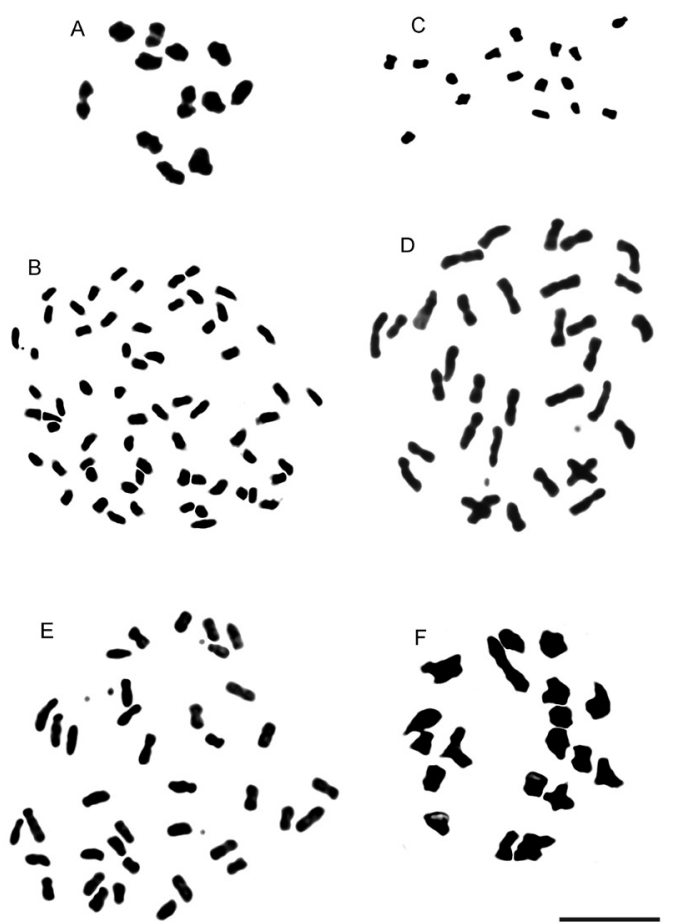

Figura 3. Cromosomas mitóticos (2n) y meióticos (n) de: A) Bidens odorata $\mathrm{n}=12$ II; B) Dahlia sorensenii $2 \mathrm{n}=64$; C) Galinsoga parviflora $2 \mathrm{n}=16$; D) Lagascea rigida $2 \mathrm{n}=34$; E) Montanoa grandiflora $2 \mathrm{n}=38$; F) M. tomentosa subsp. tomentosa $\mathrm{n}=19$ II. Escala $=10 \mu \mathrm{m}$.

número cromosómico básico x=17 sugerido para Ageratina por Watanabe et al. (1995).

Los números cromosómicos $2 \mathrm{n}=2 \mathrm{x}=18$ observados en Brickellia secundiflora (Lag.) A. Gray var. secundiflora (Fig. 2C) y en B. veronicifolia (Kunth) A. Gray (Fig. 2D) concuerdan con los registros previos para ambas especies (Turner y King, 1964; King et al., 1976). Sin embargo, difieren en el tamaño de los cromosomas, mayores en $B$. secundiflora var. secundiflora que en $B$. veronicifolia .

Con anterioridad, fueron registrados los números cromosómicos $\mathrm{n}=10$ (Sundberg et al., 1986), $\mathrm{n}=20$ (Turner et al., 1962; King et al., 1976), $\mathrm{n}=30$ (King et al., 1976) y $\mathrm{n}=40$ (Robinson et al., 1989) para Fleischmannia pycnocephala (Less.) R.M. King et H. Rob., especie ampliamente distribuida desde el suroeste de los Estados Unidos hasta Perú en Sudamérica. Tales registros muestran una serie poliploide basada en $n=10$, con poblaciones diploides, tetraploides, hexaploides y octoploides (Robinson et al., 


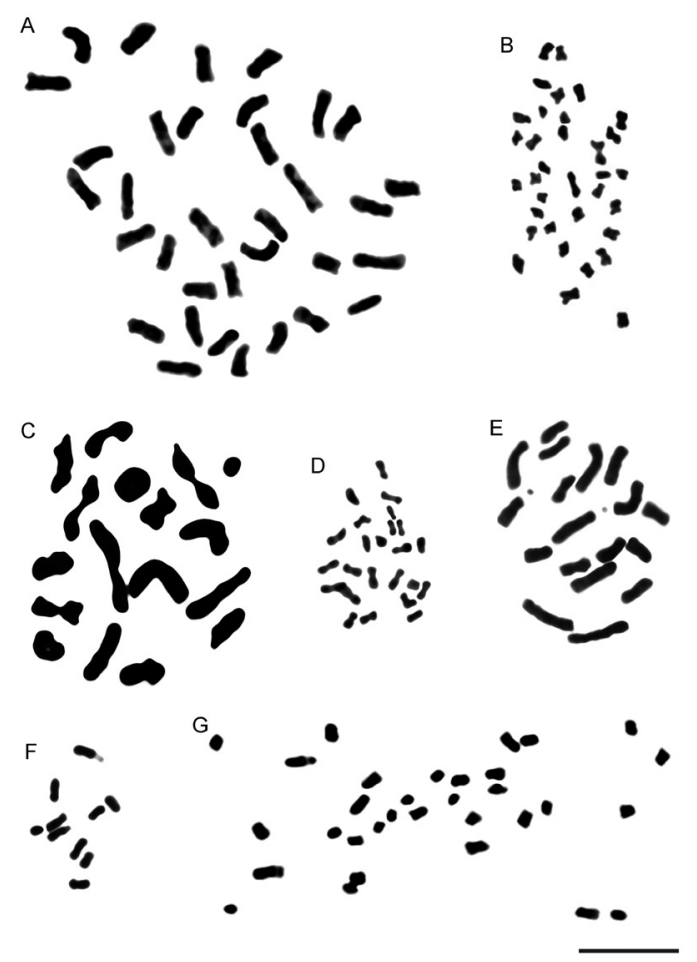

Figura 4. Cromosomas mitóticos (2n) y meióticos (n) de: A) Tithonia tubiformis $2 \mathrm{n}=34$; B) Verbesina virgata var. virgata $2 \mathrm{n}=34$; C) Viguiera buddleiformis $\mathrm{n}=17 \mathrm{II}$; D) Zinnia peruviana $2 \mathrm{n}=24$; E) Lactuca serriola $2 \mathrm{n}=18$; F) Picris echioides $2 \mathrm{n}=10$; $\mathrm{G})$ Sonchus oleraceus $2 \mathrm{n}=32$. Escala $=10 \mu \mathrm{m}$.

1989). La población analizada en este estudio es tetraploide, con $n=20$ (Fig. 2E); el nivel tetraploide es el más frecuente a lo largo de su distribución geográfica, mientras que citotipos diploides, hexaploides y octoploides tienen una frecuencia menor y distribución más restringida (Turner et al., 1962; King et al., 1976; Robinson et al., 1989).

El número cromosómico obtenido para Stevia salicifolia Cav. var. salicifolia $(2 \mathrm{n}=2 \mathrm{x}=24$, Fig. $2 \mathrm{~F})$, confirma las observaciones previas de Zhao y Turner (1993) para la misma variedad. Por otro lado, el $2 \mathrm{n}=3 \mathrm{x}=33$ observado en $S$. origanoides Kunth (Fig. $2 \mathrm{G}$ ) y $S$. tomentosa Kunth, corresponde a citotipos triploides, basados en $\mathrm{x}=$ 11 y concuerda con los registros de Watanabe et al. (2001). En estudios previos, S. origanoides, una especie morfológicamente variable y distribuida en México con amplitud, se ha mostrado la existencia de poblaciones diploides $(2 \mathrm{n}=$ $22-24)$, triploides $(2 n=33)$ y tetraploides $(2 n=44)$, siendo el citotipo triploide el más frecuente y ampliamente distribuido (Soejima et al., 2001). Las plantas triploides de
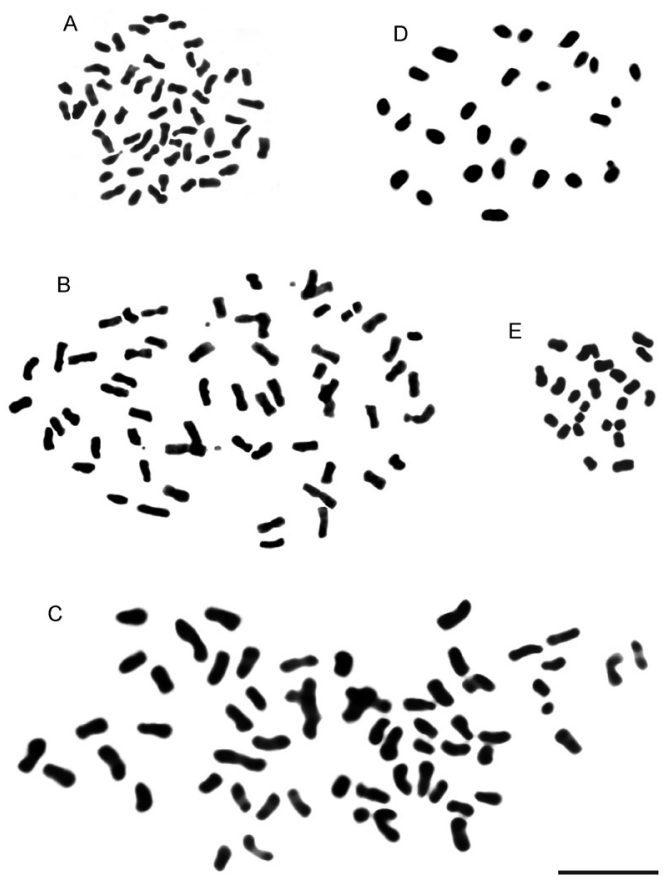

Figura 5. Cromosomas mitóticos (2n) de: A) Acourtia cordata $2 \mathrm{n}=54$; B) Barkleyanthus salicifolius $2 \mathrm{n}=60$; C) Pittocaulon praecox $2 \mathrm{n}=60$; D) Dyssodia papposa $2 \mathrm{n}=26$; E) Tagetes tenuifolia $2 \mathrm{n}=24$. Escala $=10 \mu \mathrm{m}$.

S. origanoides son estériles y su persistencia en la naturaleza depende de la propagación asexual o agamospermia (Soejima et al., 2001). Stevia es un género que incluye complejos de especies diploides y poliploides basados en $\mathrm{x}=11$ o 12; contiene más de 200 especies, distribuidas desde Norteamérica hasta Sudamérica, con su principal centro de diversificación en México (Grashoff, 1974; Watanabe et al., 2001). De acuerdo con Soejima et al. (2001) y Watanabe et al. (2001), la poliploidía acompanada por agamospermia ha contribuido a la diversificación y especiación en Stevia.

\section{Tribu Heliantheae.}

El número cromosómico $\mathrm{n}=36$ determinado para Ambrosia psilostachya DC., especie herbácea de amplia distribución en las zonas templadas de México y Norteamérica, corresponde al citotipo tetraploide, registrado de manera frecuente en el centro y norte de México (Payne et al., 1964; Keil y Stuessy, 1975). Para esta especie se han 
registrado poblaciones diploides con $\mathrm{n}=18$, tetraploides con $n=36$ y hexaploides con $n=54$ (Payne et al., 1964).

El número cromosómico $2 \mathrm{n}=2 \mathrm{x}=24$, determinado en Bidens bigelovii A. Gray var. angustiloba (DC.) Ballard y en B. odorata Cav. var. odorata (Fig. 3A) confirma conteos anteriores (Olsen, 1980; Keil et al., 1988), aunque el conteo para $B$. bigelovii var. angustiloba representa el primero para México. Ambas especies son diploides con números basados en $\mathrm{x}=12$; sin embargo, también en Bidens se han registrado especies con número básico $\mathrm{x}=10,11$ y 14 (Solbrig et al., 1972; Robinson et al., 1989).

El número cromosómico $\mathrm{n}=12$ determinado en este trabajo para Cosmos bipinnatus Cav. y C. paviflorus (Jacq.) Kunth, 2 hierbas anuales ampliamente distribuidas en México, coinciden con registros previos para ambas especies (Keil y Stuessy, 1975; Carr et al., 1999).

En Dahlia, un género con más de 35 especies distribuidas principalmente en México, se han registrado diferentes números cromosómicos, incluyendo $2 \mathrm{n}=32,34$ y 36 (Sorensen, 1969; Gatt et al., 1998). En la REPSA se encuentran 2 especies de este género, D. coccinea Cav. y D. sorensenii H.V. Hansen et Hjert. Los estudios previos en D. coccinea, una especie morfológicamente muy variable, muestran poblaciones diploides con $2 \mathrm{n}=2 \mathrm{x}=32$ (Sorensen, 1969; Gatt et al., 1998) y tetraploides con $2 n=4 x=$ 64 (Sundberg et al., 1986; Gatt et al., 1998), y al parecer las poblaciones diploides se distribuyen en áreas distintas a las ocupadas por los tetraploides (Sorensen, 1969; SotoTrejo, 2007). Las plantas analizadas aquí para $D$. coccinea corresponden a un citotipo diploide $(2 \mathrm{n}=32)$. El número cromosómico determinado en $D$. sorensenii es tetraploide $(2 n=4 x=64$; Fig. $3 B$ ), basado en $x=16$, que concuerda con los conteos hechos por Hansen y Hjerting (1996).

Se han registrado conteos cromosómicos de $n=8$ (Canne, 1983), $\mathrm{n}=16$ (Lane y Li, 1993) y $\mathrm{n}=24$ (Strother y Panero, 2001) para Galinsoga parviflora Cav., una hierba anual con gran variación morfológica a lo largo de su amplia distribución geográfica en América. El número cromosómico determinado aquí en $G$. parviflora es un diploide $2 \mathrm{n}=2 \mathrm{x}=16$ (Fig. 3C) y concuerda con los conteos para otras poblaciones del centro de México (Canne, 1983).

El número cromosómico $2 \mathrm{n}=2 \mathrm{x}=34$ (Fig. 3D) para Lagascea rigida (Cav.) Stuessy concuerda con conteos previos (Stuessy, 1978). Por otra parte, Montanoa grandiflora Alamán ex DC. con 2n= 38 (Fig. 3E) y M. tomentosa Cerv. subsp. tomentosa con $\mathrm{n}=19$ (Fig. 3F), ambas endémicas de México, son diploides basadas en $\mathrm{x}=19$ (Solbrig et al., 1972; Keil et al., 1988).

El conteo cromosómico de $2 \mathrm{n}=2 \mathrm{x}=34$ determinado para Tithonia tubiformis (Jacq.) Cass. (Fig. 4A) y para Verbesina virgata Cav. var. virgata (Fig. 4B), variedad endémica de México, concuerda con el número básico $\mathrm{x}=$ 17 ya establecido antes para ambos géneros (Turner et al., 1961).

El número cromosómico $\mathrm{n}=17$ (Fig. 4C), constituye el primer registro para Viguiera buddleiiformis, especie endémica de México. Este conteo coincide con el número básico de $\mathrm{x}=17$ establecido para el género Viguiera por Robinson et al. (1981).

El número cromosómico $2 \mathrm{n}=24$ (Fig. 4D), determinado para Zinnia peruviana (L.) L. confirma conteos previos (Torres, 1963; Lane y Li, 1993). Esta especie es diploide, con un número cromosómico basado en $\mathrm{x}=12$; sin embargo, también en Zinnia se han registrado especies con $\mathrm{x}=8,9,10$ y 11 (Solbrig et al., 1972).

Tribu Lactuceae.

El conteo cromosómico $2 \mathrm{n}=2 \mathrm{x}=18$ determinado para Lactuca serriola L. (Fig. $4 \mathrm{E}$ ) y $2 \mathrm{n}=2 \mathrm{x}=10$ para Picris echioides (Fig. 4F), 2 malezas introducidas a México provenientes de Europa y Asia, respectivamente, son los primeros registros para poblaciones del país y concuerdan con conteos precedentes (Keil y Pinkava, 1976; Tomb et al., 1978). El conteo obtenido para Sonchus oleraceus L., otra maleza introducida de Europa, confirma el número cromosómico tetraploide $2 \mathrm{n}=4 \mathrm{x}=32$ (Fig. 4G) observado por Tomb et al. (1978).

\section{Tribu Mutisieae.}

Los conteos cromosómicos $\mathrm{n}=27$ y $2 \mathrm{n}=54$ (Fig. 5A) en Acourtia cordata son los primeros para esta especie endémica de México y coinciden con los de otras especies del género, como A. carpholepis (Sch. Bip. ex A. Gray) Reveal et R.M. King (Strother y Panero, 2001), A. microcephala DC. (Vuilleumier, 1969), A. nana (A. Gray) Reveal et R.M. King (Powell y Powell, 1978), A. nudicaulis (A. Gray) B.L. Turner (Powell, et al., 1974), A. fruticosa (La Llave) B.L. Turner (registrada como $A$. rigida DC. por Carr et al., 1999), A. scapiformis (Bacig.) B.L. Turner (Carr et al., 1999), A. mexicana (Lag. ex D. Don) H. Rob. (registrada como A. thurberii (A. Gray) Reveal et R.M. King por Pinkava y Keil, 1977) y A. wrightii (A. Gray) Reveal et R.M. King (Pinkava y Keil, 1977). Powell y Sikes (1970) proponen que $\mathrm{x}=9$ podría ser el número básico en Acourtia; sin embargo, los números cromosómicos conocidos hasta el momento en 10 de las 85 especies reconocidas para el género sugieren $x=27$ como el número básico.

Tribu Senecioneae.

Barkleyanthus es un género monotípico (B. salicifolius) y constituye junto con Pittocaulon, Psacalium y 
Robinsonecio un grupo monofilético caracterizado citológicamente por tener un número cromosómico básico de $\mathrm{x}=$ 30 (Bain y Golden, 2000; Robinson et al., 1997). El conteo cromosómico diploide $2 \mathrm{n}=60$ (Fig. 5B) determinado en este estudio para $B$. salicifolius coincide con el número $\mathrm{n}=$ 30 previamente registrado (Turner et al., 1962; Turner y Flyr, 1966; Powell et al., 1974). El primer estudio cromosómico de B. salicifolius fue hecho bajo el sinónimo de Senecio salignus DC. por Turner et al. (1962).

El número cromosómico obtenido para Pittocaulon praecox fue el de un citotipo diploide $2 \mathrm{n}=60$ (Fig. 5C), basado en $\mathrm{x}=30 \mathrm{y}$ es hasta ahora la única especie estudiada citológicamente de las 5 que conforman el género (Robinson y Brettell, 1973; Strother, 1983).

\section{Tribu Tageteae.}

El conteo de $2 \mathrm{n}=2 \mathrm{x}=26$ (Fig. 5D) para Dyssodia papposa (Vent.) Hitchc., una hierba ampliamente distribuida desde Canadá hasta Guatemala, concuerda con los registros precedentes (Grashoff et al., 1972; Keil et al., 1988).

En estudios previos, Tagetes tenuifolia Cav. mostró poblaciones diploides ( $\mathrm{n}=12)$ (Keil et al., 1988) y tetrapolides $(\mathrm{n}=24)$ (Strother, 1983). El material de T. tenuifolia analizado en el presente estudio corresponde a un citotipo diploide $(2 n=2 x=24$, Fig. 5E).

Los conteos cromosómicos registrados en este trabajo incrementan el número de especies de la REPSA, para las cuales están disponibles los datos citológicos y proveen información básica relevante para investigaciones taxonómicas y evolutivas en un área natural protegida con una gran diversidad de especies de plantas.

\section{Agradecimientos}

A Enrique Ortiz por su ayuda en la recolecta del material biológico; a Javier Martínez, por su asistencia técnica en la obtención de los números cromosómicos y a Ingrid Brunner por su colaboración durante la limpieza y germinación de semillas. La primera autora agradece al Consejo Nacional de Ciencia y Tecnología (CONACYT), la beca otorgada para los estudios de posgrado (maestría) y al Posgrado en Ciencias Biológicas de la UNAM, por la oportunidad y facilidades brindadas para realizar esta investigación dentro de su programa. A PAPIIT-UNAM (Proyecto: IN214408) por el financiamiento parcial de esta investigación.

\section{Literatura citada}

Bain J. F. y J. L. Golden. 2000. A phylogeny of Packera (Senecioneae; Asteraceae) based on internal transcribed spacer region sequence data and a broad sampling of outgroups. Molecular Phylogenetics and Evolution 16:331338.

Beuzenberg, E. J. y J. B. Hair. 1984. Constributions to a chromosome atlas of the New Zealand flora- 27: Compositae. New Zealand Journal of Botany 22:353-356.

Bremer, K. 1994. Asteraceae: cladistics and classification. Timber, Portland, Oregon. 752 p.

Canne, J. M. 1983. Cytological and morphological observations in Galinsoga and related genera (Asteraceae). Rhodora 85:355-366.

Carr, D. G., R. M. King, A. M. Powell y H. Robinson. 1999. Chromosome numbers in the Compositae. XVII. American Journal of Botany 86:1003-1013.

Carrillo T. C. 1995. El Pedregal de San Ángel. Universidad Nacional Autónoma de México, México, D. F. 177 p.

Carter, C. R. 1978. The cytology of Brachycome. 8. The inheritance, frequency and distribution of B chromosomes in B. dichromosomatica $(\mathrm{n}=2)$, formerly included in $B$. lineariloba. Chromosoma 67:109-121.

Castillo-Argüero S., G. Montes-Cartas, M. A. Romero-Romero, Y. Martínez-Orea, P. Guadarrama-Chávez, I. SánchezGallen y O. Núñez-Castillo. 2004. Dinámica y conservación de la flora del matorral xerófilo de la Reserva Ecológica del Pedregal de San Ángel (D. F., México). Boletín de la Sociedad Botánica de México 74:51-75.

Castillo-Argüero, S., Y. Martínez-Orea, J. A. Meave, M. Hernández-Apolinar, O. Nuñez-Castillo, G. SantibañezAndrade y P. Guadarrama-Chávez. 2009. Flora: susceptibilidad de la comunidad a la invasión de malezas nativas y exóticas. In Biodiversidad del ecosistema del Pedregal de San Ángel, A. Lot, y Z. Cano-Santana (eds.). Universidad Nacional Autónoma de México, México D. F. p. 107-133.

Conger, A. D. y L. M. Fairchild. 1953. A quick-freeze method for making smear slides permanent. Stain Technology 28:281283.

Funk, V. A., R. J. Bayer, S. Keeley, R. Chan, L. Watson, B. Gemeinholzer, E. Schilling, J. L. Panero, B. G. Baldwin, NGarcía-Jacas, A. Susanna y R. K. Jansen. 2005. Everywhere but Antarctica: using a supertree to understand the diversity and distribution of the Compositae. Biologiske Skrifter 55:343-374.

Gaceta UNAM, 2005. Acuerdo por el que se rezonifica, delimita e incrementa la zona de la reserva ecológica del Pedregal de San Ángel de Ciudad Universitaria, Gaceta UNAM 3813:1415, 20-21, 2 de junio de 2005.

García, A. 1990. Técnicas y procedimientos de citogenética 
vegetal. Colegio de Postgraduados, Montecillo, Estado de México. 144 p.

Gatt M., H. Ding, K. Hammett y B. Murray. 1998. Polyploidy and evolution in wild and cultivated Dahlia species. Annals of Botany 81:647-656.

Grashoff, J. L. 1974. Novelties in Stevia (Compositae: Eupatorieae). Brittonia 26:347-384.

Grashoff, J. L., M. W. Bierner y D. K. Northington. 1972. Chromosome numbers in North and Central American Compositae. Brittonia 24:379-394.

Hansen, H. V. y J. P. Hjerting. 1996. Observations on chromosome numbers and biosystematics in Dahlia (Asteraceae, Heliantheae) with an account on the identity of $D$. pinnata, D. rosea, and D. coccinea. Nordic Journal of Botany 16:445455.

Keil, D. J. y D. J. Pinkava. 1976. Chromosome counts and taxonomic notes for Compositae from the United States and Mexico. American Journal of Botany 63:1393-1403.

Keil, D. J. y T. F. Stuessy. 1975. Chromosome counts of Compositae from the United States, Mexico, and Guatemala. Rhodora 77:171-195.

Keil, D. J. y T. F. Stuessy. 1977. Chromosome counts of Compositae from Mexico and the United States. American Journal of Botany 64:791-798.

Keil, D. J., M. A. Luckow y D. J. Pinkava. 1988. Chromosome studies in Asteraceae from the United States, Mexico, the West Indies, and South America. American Journal of Botany 75:652-668.

Kenton, A. 1986. Importancia de los cromosomas en la especiación y evolución como base para el conocimiento y caracterización de especies vegetales con valor potencial. In II Seminario Maximino Martínez. La aplicación de la citogenética en el conocimiento biológico de los recursos vegetales en México, G. Palomino (ed.). Jardín Botánico, Universidad Nacional Autónoma de México, México, D.F. p. 11-36.

King, R. M., D. W. Kyhos, A. M. Powell, P. H. Raven y H. Robinson. 1976. Chromosome numbers in Compositae. XIII. Eupatorieae. Annals of the Missouri Botanical Garden 63:862-888.

Lane, M. A. y J. Li. 1993. Documented chromosome numbers 1993:1. Chromosome number reports in Compositae with emphasis on tribe Astereae of the southwestern United States and Mexico. Sida 15:539-546.

Martínez, A. y J. S. Parker. 1995. Biodiversity and conservation: a role for the chromosomes. In Kew Chromosome Conference IV, P. E. Brandham y M. D. Bennett (eds.). Royal Botanic Gardens, Kew, Richmond, Surrey. p. 1-7.

Martínez, J. y G. Palomino. 1997. Evidence of heterozygous chromosome interchange and chromatid exchange in autotetraploid cytotype of Gibasis schiedeana (Tradescantieae-Commelinaceae). Cytologia 62:275-281.

Melchert, T. E. 1968. Systematic studied in the Coreopsidinae:
Cytotaxonomy of Mexican and Guatemalan Cosmos. American Journal of Botany 55:345-353.

Olsen, J. 1980. IOPB chromosome number reports LXVII. Taxon 29:346-367.

Palomino, G. 2000. Genome analysis of Mexican flora. Genetics and Molecular Biology 23:921-924.

Palomino, G. y R. Vázquez. 1991. Cytogenetic studies in Mexican populations of species of Crotalaria L. (LeguminosaePapilionoideae). Cytologia 56:343-351.

Palomino, G., B. Vázquez, P. Martínez y P. Mercado. 1990. Estudios cromosómicos en seis especies mexicanas de comelináceas. Boletín de la Sociedad Botánica de México 50:77-87.

Palomino, G., J. Dolezel, R. Cid, I. Brunner, I. Méndez y A. Rubluo. 1999. Nuclear genome stability of Mammillaria san-angelensis (Cactaceae) regenerants induced by auxins in long-term in vitro culture. Plant Science 141:191-200.

Payne, W. W., P. H. Raven y D. W. Kyhos. 1964. Chromosome numbers in the Compositae. IV. Ambrosieae. American Journal of Botany 51:418-424.

Pinkava, D. J. y D. J. Keil. 1977. Chromosome counts of Compositae from the United States and Mexico. American Journal of Botany 64:680-686.

Powell, A. M. y B. L.Turner. 1963. Chromosome numbers in the Compositae. VII. Additional species from the southwestern United States and Mexico. Madroño 17:128-140.

Powell, A. M. y S. A. Powell. 1978. Chromosome numbers in the Asteraceae. Madroño 25:160-169.

Powell, A. M. y S. Sikes. 1970. Chromosome numbers of some Chihuahuan Desert Compositae. Southwestern Naturalist 15:175-186.

Powell, A. M., D. W. Kyhos y P. H. Raven. 1974. Chromosome numbers in the Compositae. X. American Journal of Botany 61:909-913.

Ramírez, I. 1999. Comparación de cariotipos de cinco especies de dos subgéneros de Datura en México. Tesis, Facultad de Ciencias, Universidad Nacional Autónoma de México, México, D. F. 46 p.

Robinson, H. y R. D. Brettell. 1973. Studies on the Senecioneae (Asteraceae) I. A new genus, Pittocaulon. Phytologia 27:451-453.

Robinson, H., A. M. Powell, G. D. Carr, R. M. King y J. F. Weedin. 1989. Chromosome numbers in Compositae, XVI: Eupatorieae. II. Annals of the Missouri Botanical Garden 76:1004-1011.

Robinson, H., A. M. Powell, R. M. King y J. F. Weedin. 1981. Chromosome numbers in Compositae, XII: Heliantheae. Smithsonian Contributions to Botany 52:1-28.

Robinson, H., G. D. Carr, R. M. King y A. M. Powell. 1997. Chromosome numbers in Compositae XVII: Senecioneae. III. Annals of the Missouri Botanical Garden 84:893-906.

Rzedowski, J. 1954. Vegetación del Pedregal de San Ángel (D.F. México). Anales de la Escuela Nacional de Ciencias 
Biológicas, Instituto Politécnico Nacional 8:59-128.

Rzedowski, J. y G. C. de Rzedowski. 1989. Sinopsis numérica de la flora fanerogámica del Valle de México. Acta Botanica Mexicana 8:15-30.

Rzedowski, J. y G. C. de Rzedowski. 1993. Datos sobre la dinámica de la flora fanerogámica del Valle de México, con énfasis en las especies nativas raras, en peligro de extinción y aparentemente extintas. Acta Botanica Mexicana 25:81-108.

Rzedowski, J. y G. C. de Rzedowski. 2001. Flora fanerogámica del Valle de México. Comisión Nacional para el Conocimiento y Uso de la Biodiversidad/Instituto de Ecología, A.C. Pátzcuaro, Mich. 1406 p.

Semple, J. C. y K. Watanabe. 2009. A review of chromosome numbers in Asteraceae with hypothesis on chromosomal base number evolution. In Systematics, evolution, and biography of Compositae, V. A. Funk, A. Susanna, T. F. Stuessy y R.J. Bayer (eds.). IAPT, Vienna. p. 61-72.

Soejima, A., T. Yahara y K. Watanabe. 2001. Distribution and variation of sexual and agamospermous populations of Stevia (Asteraceae: Eupatorieae) in the lower latitudes, Mexico. Plant Species Biology 16:91-105.

Solbrig, O. T. 1977. Chromosomal cytology and evolution in the family Compositae. In The biology and chemistry of the Compositae, vol.1, V. H. Heywood, J. B. Harborne y B. L. Turner (eds.) Academic, London.. p. 267-281.

Solbrig, O. T., D. W. Kyhos, A. M. Powell y P. H. Raven. 1972. Chromosome numbers in the Compositae. VIII. Heliantheae. American Journal of Botany 59:869-878.

Sorensen, P. D. 1969. Revision of the genus Dahlia (Compositae, Heliantheae, Coreopsidinae). Rhodora 71:309-416.

Soto-Trejo, F. 2007. Poliploidía en las Asteraceae en la Reserva Ecológica del Pedregal de San Ángel, México, D.F. Tesis Maestría, Facultad de Ciencias, Universidad Nacional Autónoma de México, México, D.F. 85 p.

Strother, J. L. 1983. More chromosome studies in Compositae. American Journal of Botany 70:1217-1224.

Strother, J. L. y J. L. Panero. 2001. Chromosome studies: Mexican Compositae. American Journal of Botany 88:499-502.

Stuessy, T. F. 1978. Revision of Lagascea (Compositae, Heliantheae). Fieldana, Botany 38:75-133.

Stuessy, T. F., H. Weiss-Schneeweiss y D. J. Keil. 2004. Diploid and polyploid cytotype distribution in Melampodium cinereum and M. leucanthum (Asteraceae, Heliantheae). American Journal of Botany 91:889-898.

Sundberg, S., C. P. Cowan y B. L. Turner. 1986. Chromosome counts of Latin American Compositae. American Journal of Botany 73:33-38.

Tomb, S. A., K. L. Chambers, D. W. Kyhos, A. M. Powell y P. H. Raven. 1978. Chromosome numbers in the Compositae. XIV. Lactuceae. American Journal of Botany 65:717-721.

Torres, A. M. 1963. Taxonomy of Zinnia. Brittonia 15:1-25.
Turner, B. L., A. M. Powell, T. J. Watson. 1973. Chromosome numbers in Mexican Asteraceae. American Journal of Botany 60:592-596.

Turner, B. L. y D. Flyr. 1966. Chromosome numbers in the Compositae. X. North American species. American Journal of Botany 53:24-33.

Turner, B. L. y G. Nesom. 1998. Biogeografía, diversidad y situación de peligro o amenaza de Asteraceae en México. In Diversidad biológica de México: orígenes y distribución, T. P. Ramamoorthy, R. Bye, A. Lot y J. Fa (eds.). Instituto de Biología, Universidad Nacional Autónoma de México, México, D.F. p. 545-561.

Turner, B. L. y M. C. Johnston. 1961. Chromosome numbers in the Compositae-III. Certain Mexican species. Britonia 13:64-69.

Turner, B. L. y R. M. King. 1964. Chromosome numbers in the Compositae. VIII. Mexican and Central American species. Southwestern Naturalist 9:27-39.

Turner, B. L., M. Powell y R. M. King. 1962. Chromosome numbers in the Compositae. VI. Additional Mexican and Guatemalan species. Rhodora 64:251-269.

Turner, B. L., W. L. Ellison y R. M. King. 1961. Chromosome numbers in the Compositae. IV. North American species, with phyletic interpretations. American Journal of Botany 48:216-223.

Villaseñor, J. L. 2003. Diversidad y distribución de las Magnoliophyta de México. Interciencia 28:160-167.

Villaseñor, J. L., E. Ortiz y V. Juárez. 2004. Asteráceas. In Biodiversidad de Oaxaca, A. J. García, M. J. Ordóñez y M. Briones-Salas (eds.). Instituto de Biología, UNAM/Fondo Oaxaqueño para la Conservación de la Naturaleza/World Wildlife Fund, México, D.F. p. 177-192.

Vuilleumier, B. S. 1969. The systematics and evolution of Perezia Sect. Perezia (Compositae). Contributions of the Gray Herbarium 199:3-163.

Watanabe, K., T. Yahara, T. Denda y K. Kosuge. 1999. Chromosomal evolution in the genus Brachyscome (Asteraceae, Astereae): statistical tests regarding correlation between changes in karyotype and habit using phylogenetic information. Journal of Plant Research 112:145-161.

Watanabe, K., R. M. King, T. Yahara, M. Itos, J. Yokoyama, T. Suzuki y D. J. Crawford. 1995. Chromosomal cytology and evolution in Eupatorieae (Asteraceae). Annals of the Missouri Botanical Garden 8:2581-2592.

Watanabe, K., T. Yahara, A. Soejima y M. Ito. 2001. Mexican species of the genus Stevia (Eupatorieae, Asteraceae). Chromosome numbers and geographical distribution. Plant Species Biology 16:49-68.

Zhao, Z. y B. L. Turner. 1993. Documented chromosome numbers 1993: 3. Miscellaneous U.S.A. and Mexican species, mostly Asteraceae. Sida 15:649-653. 
